\title{
Perfusion-based Brain Connectivity: PASL vs pCASL
}

\author{
Bianca De Blasi \\ Dept. of Medical Physics \\ University College London \\ London, UK \\ bianca.blasi.15@ucl.ac.uk \\ Gloria Menegaz \\ Dept. of Computer Science \\ University of Verona \\ Verona, Italy \\ gloria.menegaz@univr.it
}

\author{
Anna Barnes \\ Inst. of Nuclear Medicine \\ University College London \\ Hospitals \\ London,UK \\ anna.barnes1@nhs.net \\ Enrico De Vita \\ Dept. of Biomedical \\ Engineering \\ King's College London, \\ London, UK \\ enrico.devita@kcl.ac.uk
}

\author{
Silvia Francesca Storti \\ Dept. of Computer Science \\ University of Verona \\ Verona, Italy \\ silviafrancesca.storti@univr.it
}

\author{
Ilaria Boscolo Galazzo \\ Dept. of Computer Science \\ University of Verona \\ Verona, Italy \\ ilaria.boscologalazzo@univr.it
}

\author{
Matthias Koepp \\ Inst. of Neurology \\ University College London \\ London, UK \\ m.koepp@ucl.ac.uk
}

\begin{abstract}
Arterial Spin Labelling (ASL) is a magnetic resonance imaging technique which provides a more direct measure of neural activity as compared to blood-oxygenationlevel-dependent (BOLD) contrast. While it has been used for years for perfusion quantification, ASL has recently been adopted for functional connectivity (FC) analyses. However, the impact of the different ASL schemes on connectivity estimates remains to be fully investigated. In this work, pulsed and pseudo continuous ASL (PASL/pCASL) were compared in terms of cerebral blood flow (CBF) and FC measures. In line with literature, higher $\mathrm{CBF}$ and increased spatial signal-to-noise ratio were reported for $\mathrm{PCASL}$, as compared to PASL. In terms of FC, pCASL was able to more reliably recover the main networks and showed higher correlations between brain areas. These preliminary results suggest pCASL to provide reliable and stable results, not only for CBF estimation but also for FC analyses.
\end{abstract}

Keywords-ASL, PASL, pCASL, CBF, Functional
Connectivity

\section{INTRODUCTION}

Arterial Spin Labelling (ASL) is a magnetic resonance imaging (MRI) technique which allows the non-invasive quantification of brain perfusion [1]. Three main ASL sequences have been developed during the last years which exploit different ways to magnetically label blood water as it flows to the brain. Pulsed ASL (PASL) uses a short inversion pulse to tag a thick slab of blood spins passing through the neck [2]. Continuous ASL (CASL) uses an adiabatic inversion pulse to label blood flowing through a single plane at the neck level [2]. Despite providing increased signal-to-noise ratio (SNR), its use has been hampered by a high radio frequency duty cycle. Pseudo-continuous ASL (pCASL) was introduced as an alternative to CASL which can be implemented on standard MRI RF amplifiers. It involves blood labelling by a train of discrete RF-pulses which mimic the CASL tagging, while being less demanding in terms of duty cycle and energy deposition [3].

The impact of these labelling schemes in the estimation of cerebral blood flow (CBF) has been investigated in several recent works [3-5]. In particular, Chen and colleagues [3] assessed the reproducibility of PASL, CASL and pCASL with 2D-readout in a group of healthy controls. They reported higher SNR as well as higher reproducible and precise CBF measurements for pCASL compared to the other methods.
Besides CBF estimation, ASL has recently been adopted to derive functional connectivity (FC) measures, as an alternative technique to the well-known and more established blood-oxygenated-level-dependent (BOLD) functional MRI (fMRI). This is because the latter is an indirect measure of the neural activity, resulting from the overall contribution of CBF, blood volume and metabolism, and it is highly hampered by vein contamination. Conversely, ASL signals can provide more direct FC measures.

FC is currently estimated using different methods [6], with independent component analysis (ICA) and seed-based analysis being the most common. ICA is a data-driven method which aims at separating the data into spatially and temporally independent components (ICs). These components represent either functionally relevant networks, namely resting-state networks (RSNs), or noise and artefacts. Seed-based analysis involves the a priori definition of regions of interest (ROIs). Average time series are extracted from those regions and their coupling, usually expressed in terms of correlation, represents the FC strength between the selected brain areas. Few studies have started to investigate FC based on ASL [7-9]. Jann et al. [8] and Dai et al. [9] showed that ASL can lead to functional networks similarly to BOLD fMRI, using different methodologies applied to a motor task and resting-state data, respectively.

As in the case of CBF, it is important to understand how FC estimates might change with different ASL sequences. However, in this context it is difficult to define an appropriate ground truth as to how FC should look like. Nevertheless, assessing how FC results change by using different ASL techniques, which are characterised by different tagging, specific acquisition parameters and temporal resolution, remains timely and important. Some works compared the perfusion-based connectivity measures obtained from pCASL with 2D and 3D readouts [11-12]. In particular, Jann et al. [12] compared 3D-GRASE and 2D-echo planar imaging (EPI) pCASL in terms of SNR and FC results. They reported improved temporal SNR (tSNR) of the 3D readout which allowed more reliable perfusion-based $\mathrm{FC}$ as compared to 2D readout. They also showed that the $2 \mathrm{D}$ readout can provide perfusion images with high contrast but its lower tSNR might hamper reliable FC estimation.

However, to the best of our knowledge, the possible differences in both CBF and FC metrics derived from PASL and pCASL sequences with the same 2D-EPI readout have not 
been previously investigated. Therefore, in this study we aimed to provide a comparison of these two ASL techniques in terms of not only CBF maps, but also FC measures.

\section{METHODS}

\section{A. Population and Image Acquisition}

This study was approved by the local Ethics Committee (REC: $15 / \mathrm{LO} / 1051$ ). Five healthy subjects ( 3 males, $37 \pm 2 \mathrm{y}$, all right-handed) provided written informed consent and were enrolled. Imaging was carried out on a 3T Siemens PET/MR scanner equipped with a 16-channel head and neck coil.

Each subject was scanned with both PASL and pCASL sequences during a unique session on the PET/MR scanner while awake and in resting-state (eyes-closed). For PASL, the Siemens' PICORE (proximal inversion with a control for offresonance effects) sequence with Q2TIPS (QUIPSS II with Thin-slice TI1 Periodic Saturation) and 2D-EPI readout was used [voxel size: $3.6 \times 3.6 \times 5 \mathrm{~mm}^{3}$; gap: $1 \mathrm{~mm} ; 19$ partitions; TI1/TIs/TI2: 800/1200/1800 ms; TR/TE: $2860 / 17 \mathrm{~ms}] .200$ Control/Label volumes, plus a calibration scan $\left(\mathrm{M}_{0}\right)$ with long TR were acquired (total scanning time: 9'39"). The pCASL sequence was provided by Dr JJ Wang from UCLA [13]. This version was installed and optimised in our Center in order to make it as much similar as possible to the PASL one. In particular, the same spatial resolution as for the PASL was kept, while the other parameters were changed as follows: bolus duration/PLD: $1800 / 1800 \mathrm{~ms}$; TR/TE: $4590 / 17 \mathrm{~ms}$. The number of Control/Label volumes was reduced to 124 in order to keep the same total scanning time.

A 3D T1-weighted (T1w) MPRAGE anatomical scan was finally included for segmentation and registration purposes [voxel size: $1.1 \times 1.1 \times 1.1 \mathrm{~mm}^{3}$; 208 sagittal partitions, TR/TE: 2000/2.92 ms].

\section{B. ASL Pre-processing}

Analysis of ASL data was carried out using FSL 5.0.9 (FMRIB, Oxford, UK). PASL and pCASL data were analysed separately. The pre-processing steps to obtain clean ASL data included: 1) motion correction (MCFLIRT) using $\mathrm{M}_{0}$ as reference; 2 ) regression of the 6 motion parameters (3 translation +3 rotations) estimated during step 1 . These preprocessing steps are commonly adopted for ASL [12].

\section{CBF Estimation}

Having obtained clean ASL datasets for each subject, we computed CBF maps at the single subject level. ControlLabel difference images $(\Delta M)$ were calculated pair-wise and visually/qualitatively evaluated: those with marked artefacts (e.g. severe ringing around the brain due to residual motion), were discarded from further analysis.

To compare the two acquisition protocols, spatial SNR was computed on the $\Delta \mathrm{M}$ images, calculating representative values over grey matter (GM [sSNR-GM]) and white matter (WM [sSNR-WM]) [11]. The sSNR was calculated as the ratio of the mean $\triangle \mathrm{M}$ in each tissue and the standard deviation (STD) of the noise (sampled in a square ROI in the background area around the brain). To do this, each subject's structural image was segmented to obtain tissue probability maps for GM and WM, which were then thresholded at 0.9 and binarized. These masks were back-projected in ASL space by inverting the linear registration from $\mathrm{M}_{0}$ (ASL reference) to the corresponding T1w image (Boundary-Based Registration, ASL-to-T1).
CBF maps were computed for each subject using BASIL FSL toolbox ${ }^{\mathrm{a}}$. Default relaxation values were used (tissue T1: $1.3 \mathrm{~s}$, arterial T1: $1.65 \mathrm{~s}$ ). Equilibrium blood magnetisation was estimated voxel-wise by using the $\mathrm{M}_{0}$ image [2]. In order to register the $\mathrm{CBF}$ maps to standard space, the transformation of the T1w image to the 2-mm MNI standard space was estimated using an initial linear step (FLIRT, 12 degrees of freedom), followed by a non-linear step (FNIRT) [T1-to-MNI]. The ASL-to-T1 and T1-to-MNI transformations were combined and finally applied to the individual $\mathrm{CBF}$ images.

Group CBF maps were derived separately for PASL and pCASL. Additionally, we computed the coefficient of variation $(\mathrm{CV})$ defined as the ratio of the group STD and mean, for each sequence. To provide representative CBF values for PASL and pCASL, we calculated the mean CBF across GM and WM masks for each subject in native space. The Wilcoxon signed rank test was applied to statistically compare the CBF values from the two sequences $(p<0.05)$. Additionally, we computed the ratio of mean CBF in GM over WM as a measure of contrast ratio for each sequence $[4,11]$.

\section{FC Measures - Group ICA}

After spatially normalizing the clean ASL volumes using the combined transformation previously estimated, a group ICA was run (multisession temporal concatenation, MELODIC FSL). The number of ICs was fixed to 40 [14]. The most common RSNs described in the literature, including default mode network (DMN), motor, visual (occipital, medial and lateral), auditory, frontal-parietal (left and right) and cerebellum networks, were visually identified and compared to the BOLD template [15]. Of note, this template was considered as reference in this study, despite being based on the BOLD fMRI data, as no ASL derived templates are currently available in the literature. The cross-correlation (rvalue) was computed between each IC map and the corresponding BOLD template (FSL tool $f s l c c$ ). Our hypothesis was that the more the group ICA maps correlated with the corresponding templates the better the true signal was identified [15].

\section{E. FC Measures - ROI-to-ROI Analysis}

In order to estimate the ROI-to-ROI connectivity and derive FC matrices at the single-subject level, the functional Schaefer atlas with 17 RSNs and 100 ROIs was used ${ }^{b}$. Starting from the clean ASL data in standard space, the average time series were extracted from each ROI and the full correlation computed between all pairs of regions, leading to two FC matrices, one per sequence. The overall mean and STD matrices were then computed across subjects, separately for PASL and pCASL. The former was reported as a summary of the FC estimated from each ASL sequence. The latter was calculated as we expect the variability across our homogenous group of healthy subjects to decrease when the connectivity is estimated with more stable methods [16].

\section{RESULTS}

\section{A. CBF Maps}

Fig. 1 reports the mean $\mathrm{CBF}$ and $\mathrm{CV}$ maps calculated across the five subjects, for both PASL and pCASL. Higher values of perfusion are reported for pCASL, while PASL shows reduced values across the whole brain. 
Lower CV values were found for pCASL as compared with PASL, especially across the GM.

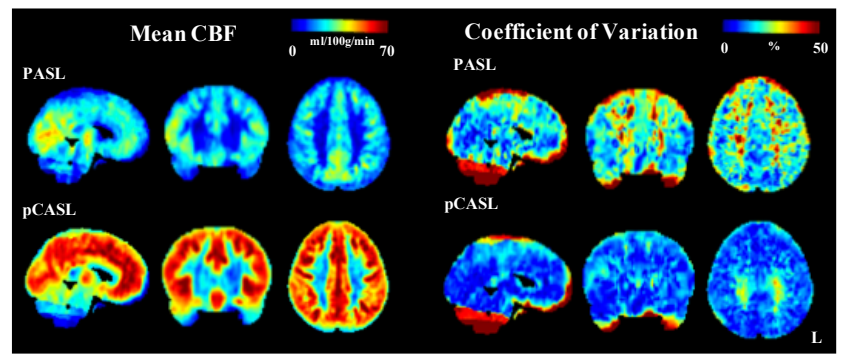

Fig. 1. Group cerebral blood flow (CBF) and coefficient of variation (CV) maps calculated across subjects for PASL and pCASL.

Table I reports the summary measures for sequence comparison. PCASL showed statistically higher values in both tissue types ( $\mathrm{p}=0.0312$, one-tailed) compared to PASL. The latter appeared to underestimate perfusion in GM, also in comparison to literature values. Of note, for PASL data, on average $49 \%$ of $\Delta \mathrm{M}$ was eliminated per subject, due to severe artefacts that were corrupting the $\Delta \mathrm{M}$ maps. Increased sSNR was found in both GM and WM for pCASL when compared to PASL.

TABLE I. $\quad$ MEAN \pm STD CBF (ML/100G/MIN), GM-WM CONTRAST RATIO AND SSNR

\begin{tabular}{|c|c|c|}
\hline & PASL & pCASL \\
\hline Mean CBF GM & $28.8 \pm 3.8$ & $51.9 \pm 7.0$ \\
\hline Mean CBF WM & $17.9 \pm 2.9$ & $31.6 \pm 5.4$ \\
\hline GM-WM Contrast Ratio & $1.6 \pm 0.2$ & $1.7 \pm 0.2$ \\
\hline sSNR GM & $22.4 \pm 10.7$ & $28.8 \pm 9.2$ \\
\hline sSNR WM & $8.3 \pm 3.5$ & $13.8 \pm 3.6$ \\
\hline
\end{tabular}

\section{B. FC Measures - Group ICA}

Table II reports the r-value between each IC map and the corresponding BOLD template. In literature, a correlation of $r>=0.25$ is generally considered as a valuable cut-off value for classifying a good component from BOLD fMRI. Therefore, our results pointed towards a general strong correlation between maps from ASL and BOLD fMRI. Overall, pCASL showed increased correlation with the template and seemed to outperform in high-level cognitive networks such as DMN (0.32 PASL vs 0.68 pCASL) and frontal parietal networks, which were not recovered by the PASL sequence. On the other hand, sensory networks such as motor, auditory and visual were equally represented by both sequences.

TABLE II. CROSS-CORRELATION (R-VALUE) BETWEEN THE GROUP ICA MAPS AND CORRESPONDING BOLD FMRI TEMPLATE. NA: NOT AVAILABLE

\begin{tabular}{|l|c|c|}
\hline & PASL & pCASL \\
\hline DMN & 0.32 & 0.68 \\
\hline Motor & 0.56 & 0.56 \\
\hline Medial Visual & 0.67 & 0.77 \\
\hline Lateral Visual & 0.34 & 0.48 \\
\hline Occipital Visual & 0.44 & 0.26 \\
\hline Auditory & 0.34 & 0.34 \\
\hline Frontal Parietal L & NA & 0.49 \\
\hline Frontal Parietal R & NA & 0.35 \\
\hline Cerebellum & 0.41 & 0.42 \\
\hline
\end{tabular}

Fig. 2 reports the group DMN and medial visual maps, as indicative examples of the perfusion-based networks derived by the two different ASL protocols. It can be visually appreciated that pCASL recovered the DMN more accurately, especially the PCC region, and had generally higher connectivity values across the network.

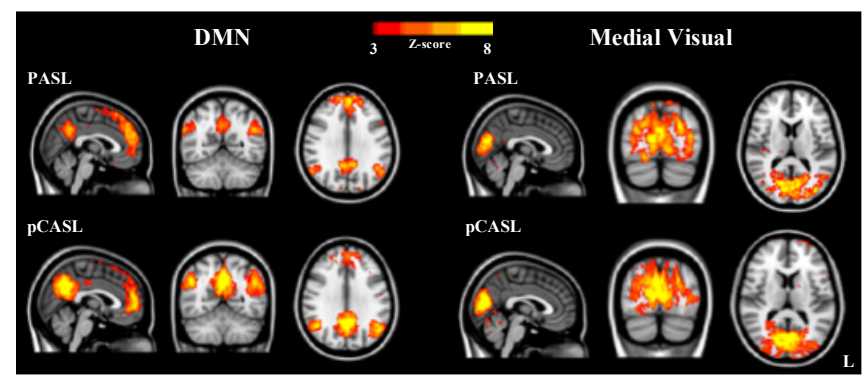

Fig. 2. Group DMN and medial visual maps for PASL and pCASL. The same slices in MNI space are reported with a threshold of $z>3$.

\section{FC Measures - ROI-to-ROI Analysis}

Fig. 3 reports the FC matrices derived for each subject and sequence along with the corresponding overall mean and STD matrices. The spatial correlation between individual PASL and pCASL matrices was $0.64 \pm 0.07$ (mean \pm STD across subjects). Globally, pCASL revealed higher FC values, compared to PASL, as visible in all subjects. Considering the overall mean and STD matrices, the mean (mean STD) values were $0.51(0.130)$ and $0.58(0.126)$ for PASL and pCASL, respectively.

\section{DISCUSSION}

This preliminary study investigates the main differences between PASL and pCASL datasets in a group of healthy subjects, in terms of both CBF maps and perfusion-based FC. ASL is a relatively new functional imaging modality which has been shown to provide a more direct measure of neural activity as compared to BOLD [3]. Reproducible CBF measures have been reported in literature from ASL data, with results comparable to positron emission tomography [2]. Besides its use for perfusion quantification, ASL has been shown to provide good localisation results and reliable connectivity estimates in both task and resting-state data, given its more direct and quantifiable signal [8]. However, over the past year several ASL sequences, characterised by different tagging and read-out methods, have been developed. It is therefore essential to assess if and how FC patterns change when using different ASL acquisition schemes.

In terms of $\mathrm{CBF}$, our results are in line with previous studies comparing PASL and pCASL [3]. We reported a statistically significant difference between the CBF estimated with the two sequences $(p<0.05)$. In particular, we found higher values (in the range of those reported in literature for both GM and WM) for pCASL as compared to PASL, which tends to underestimate the CBF in GM. However, the GM-toWM contrast ratios are similar to those reported in Dolui et al. [4], thereby confirming that both sequences are able to estimate $\mathrm{CBF}$ with comparable contrast.

In terms of FC and RSN, we found that pCASL was able to recover the main networks with higher consistency with respect to BOLD, than PASL. Indeed, we reported increased cross-correlation with the template for most of the networks, especially those involved in high-level cognitive functions. In particular, some networks (frontal-parietal left and right) 


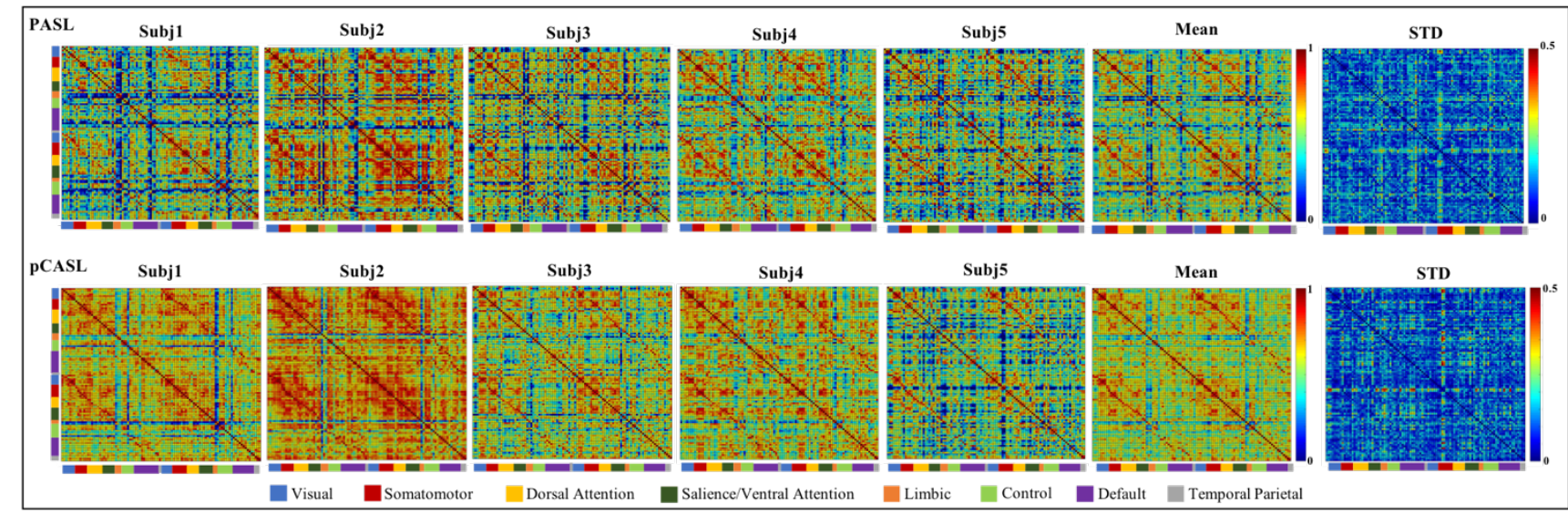

Fig. 3. Individual connectivity matrices plus the corresponding overall mean and STD matrices across subjects for each sequence.

were only recovered by the pCASL. Regarding FC and ROIto-ROI analysis, connectivity matrices showed increased mean connectivity when pCASL was used and decreased within-group STD. This finding is important as it hints to the fact that FC measures from pCASL might be more stable across subjects, as expected in a healthy population. In addition, it can be noted that the FC patterns are consistent when PASL and pCASL are compared for a given subject. However, single-subject pCASL connectivity matrices generally show increased values when compared to PASL, which was also confirmed by the slightly increased mean connectivity across subjects.

As a general limitation, we want to emphasise the lack of an absolute ground truth of FC strength and patterns. This extends to the whole FC literature [16], as gold standard FC matrices are currently lacking and also simulating reasonable scenarios might be challenging, especially in the case of ASL. This makes it difficult to objectively compare different acquisition strategies. However, investigating how FC patterns are modulated by the ASL acquisition type is timely and important, as it can provide insights on how FC derived from ASL changes in relation to different data resolution and tagging properties of a given sequence. In addition to this, we acknowledge the preliminary stage of this work where only a limited sample size was analysed. Despite the limited sample size, we were able to demonstrate changes in both CBF and FC patterns with different ASL sequences. pCASL achieved more reliable results in terms of both $\mathrm{CBF}$ and $\mathrm{FC}$ estimations, while PASL tended to underestimate perfusion and fails to recover some of the most common networks. In summary, this preliminary work shows promising results in terms of perfusion-based connectivity which can help underpinning the functional organisation of brain networks, using a more direct measure of neural activity.

\section{AKNOWLEDGMENT}

This project is supported by the EPSRC-funded UCL Centre for Doctoral Training in Medical Imaging (EP/L016478/1) and the Department of Health's NIHRfunded Biomedical Research Centre at UCLH. Additionally, this work is supported by the collaboration between UCL (UK) and the Department of Computer Science in the University of Verona (Italy).

\section{REFERENCES}

[1] Y. Chen, D. J. J. Wang and J. A. Detre, "Comparison of arterial transit times estimated using arterial spin labeling," Magn. Reson. Mater. Phy., vol. 25, pp. 135-144, 2012.

[2] D. C. Alsop, et al. "Recommended implementation of arterial spinlabeled perfusion MRI for clinical applications: a consensus of the ISMRM perfusion study group and the european consortium for ASL in dementia," Magn. Reson. in Medicine, vol. 73, pp. 102-116, 2015.

[3] Y. Chen, D. J. J. Wang and J. A. Detre, "Test-retest reliability of arterial spin labeling with common labeling strategies," Journal of Magnetic Resonance Imaging, vol. 33, pp. 940-949, 2011.

[4] S. Dolui, et al., "Comparison of PASL, PCASL, and backgroundsuppressed 3D PCASL in mild cognitive impairment," HBM, vol. 38, pp. 5260-5273, 2017.

[5] D. Ivanov, et al., "Comparison of $3 \mathrm{~T}$ and $7 \mathrm{~T}$ ASL techniques for concurrent functional perfusion and BOLD studies," NeuroImg, vol. 156, pp. 363-376, 2017.

[6] M. P. van den Heuvel and H. E. H. Pol, "Exploring the brain network: a review on resting-state fMRI functional connectivity," European Neuropsychopharmacology, vol. 20, pp. 519-534, 2010.

[7] I Boscolo Galazzo, et al., "Arterial spin labelling reveals distrupted brain networks and functional connectivity in drug-resistant temporal epilepsy," Front. Neuroinf., vol. 12, 2019.

[8] K. Jann, et al., "Quantification of network perfusion in ASL cerebral blood flow data with seed based and ICA approaches," Brain. Topogr., vol. 26, pp. 569-580, 2013.

[9] W. Dai, G. Varma, R. Scheidegger and D. C. Alsop, "Quantifying fluctuations of resting state networks using arterial spin labeling perfusion MRI,” JCBFM, vol. 36, pp. 463-473, 2016.

[10] S.F Storti, I. Boscolo Galazzo, F. B. Pizzini, G. Menegaz, "Dual-echo ASL based assessment of motor networks: a feasibility study," J. Neural. Eng., vol. 15, pp. 1-19, 2018.

[11] M. Vidoretta, et al., "Comparison of 2D and 3D single-shot ASL perfusion fMRI sequences," NeuroImg, vol. 0, pp. 662-671, 2013.

[12] K. Jann, R. X. Smith, E. A. R. Piedra, M. Dapretto, D. J. J. Wang, "Noise reduction in arterial spin labeling based functional connectivity using nuisance variables," Frontiers in Neuroscience, vol. 10, pp. 1-13, 2016

[13] D. J. J. Wang, et al., "The value of arterial spin-labeled perfusion imaging in acute ischemic stroke - comparison with dynamic susceptibility contrast enhanced MRI," Stroke, vol. 43, pp. 1018-1024, 2012.

[14] Z. Liang, J. King and N. Zhang, "Uncovering intrinsic connectional architecture of functional networks in awake rat brain," The Journal of Neuroscience, vol. 31, pp. 3776-3783, 2011.

[15] S. M. Smith, et al., "Correspondence of the brain's functional architecture during activation and rest," PNAS, vol. 106, pp. 13040$13045,2009$.

[16] L. Griffanti, et al., "Effective artefact removal in resting state fMRI data improves detection of DMN functional connectivity alteration in Alzheimer's disease," Frontiers in Human Neuroscience, vol. 9, pp. 1$11,2015$. 